\title{
La neurofisiología clínica: pasado, presente y futuro
}

\section{Clinical Neurophysiology: past, present, future}

\author{
G. Morales ${ }^{1}$, J. Artieda ${ }^{2}$
}

\section{RESUMEN}

La Neurofisiología Clínica es una especialidad médica cuyo objeto es el estudio del sistema nervioso y muscular con fines diagnósticos, pronósticos y terapéuticos.

En este artículo se analiza el objetivo básico que pretende esta disciplina, las técnicas que utiliza y su reconocimiento como especialidad médica. Se hace un pequeño recorrido por su definición y alcance de la misma, cómo se estructura hoy día y las posibilidades de futuro que ofrece.

Palabras clave. Neurofisiología Clínica. Electroencefalografía. Electromiografia. Potenciales evocados. Sueño.

\begin{abstract}
Clinical Neurophysiology is a medical speciality whose aim is the study of the nervous and muscular system for diagnostic, prognostic and therapeutic purposes.

This article analyses the basic objective pursued by this discipline, the techniques it employs and its recognition as a medical speciality. The article briefly reviews its definition and scope, how it is structured at present and the future possibilities it offers.
\end{abstract}

Key words. Clinical Neurophysiology. Electroencephalography. Electromyography. Evoked potentials. Sleep.
1. Servicio de Neurofisiología Clínica.

Hospital Virgen del Camino. Pamplona

2. Servicio de Neurofisiología Clínica. Área de Neurociencias. CIMA y

Clínica de la Universidad de Navarra.

Pamplona.

\section{Correspondencia}

Gonzalo Morales Blánquez

Servicio de Neurofisiología Clínica

Hospital Virgen del Camino

Irunlarrea, 4

31008 Pamplona 


\section{INTRODUCCIÓN}

La Neurofisiología Clínica es una disciplina médica cuyo objetivo básico es el estudio fisiopatológico de los grandes síndromes y enfermedades del sistema nervioso central y periférico, incluido el componente nervioso de los órganos sensoriales y el sistema muscular. Utiliza técnicas instrumentales basadas fundamentalmente en el registro de la actividad eléctrica o magnética de distintas estructuras o sistemas, en situación basal o tras su estimulación. Esta definición es un poco más amplia que la expresada en el BOE 224 de 16 de septiembre de 2008 que define a la Neurofisiología Clínica ${ }^{1}$ como una especialidad médica que se fundamenta en los conocimientos de las neurociencias básicas y tiene como objetivo la exploración funcional del sistema nervioso, utilizando las técnicas de electroencefalografía, de electromiografía, de polisomnografía, de potenciales evocados, de magnetoencefalografía, así como de neuromodulación, con fines diagnósticos, pronósticos y terapéuticos. Estas técnicas neurofisiológicas son claves en el diagnóstico, cuantificación y seguimiento de distintas enfermedades neurológicas, de los órganos de los sentidos y del músculo. Su conocimiento es de utilidad en el ejercicio de distintas especialidades como la Medicina Interna, Neumología, Neurocirugía, Neurología, Pediatría, Psiquiatría, Rehabilitación, Reumatología, Otorrinolaringología, Oftalmología, Traumatología, Medicina Intensiva, Medicina Legal, Medicina del Trabajo, etc. Todas ellas demandantes de exploraciones neurofisiológicas lo que confiere a esta especialidad el carácter de servicio central.

La Neurofisiología tiene una larga tradición en España ya que el primer electroencefalógrafo se instaló en Burgos en al año 1937 como soporte para el diagnóstico en neurocirugía. En la década de los 40 surgen unidades específicas de electroencefalografía en varios hospitales bajo responsabilidad de médicos procedentes de distintas áreas como la neurología, la pediatría y la psiquiatría. En el año 1958 dichas unidades ya están presentes en hospitales clínicos de Barcelona, Madrid, Pamplona y Santiago de Compostela donde se impartían programas de formación y cursos monográficos de doctorado. Todo ello hizo que las unidades se fueran difundiendo por toda España al tiempo que incorporaban nuevas técnicas como la electromiografía, los potenciales evocados, la polisomnografía etc, de forma que en el año 1977, 128 hospitales públicos tenían servicios centrales de Neurofisiología Clínica.

La creación y consolidación de la Neurofisiología Clínica como especialidad médica independiente ha quedado plasmada desde el inicio en las distintas disposiciones que han regulado las especialidades médicas en nuestro país concretamente en los RD 2015/1978, 127/1984 y 183/2008. El periodo de formación de esta especialidad en el sistema MIR es de 4 años durante los cuales el futuro especialista además de adquirir una formación en neurociencias y en las enfermedades del sistema nervioso y de los órganos sensoriales, deberá cualificarse en las cuatro áreas fundamentales de la Neurofisiología Clínica, es decir la Electroencefalografía (EEG), la Electromiografía (EMG) la Polisomnografía (PSG) y los Potenciales Evocados (PE). Asimismo la Neurofisiología Clínica tiene rango de especialidad médica independiente en diversos países de Europa (Dinamarca, Suecia, Finlandia, Noruega y Reino Unido) y figura reconocida como tal en la Directiva 2005/36/ CE del Parlamento Europeo y del Consejo, relativa al reconocimiento de las cualificaciones profesionales.

\section{LA NEUROFISIOLOGÍA CLÍNICA: PRESENTE Y FUTURO}

Los servicios de Neurofisiología Clínica de primera línea deben tener tres áreas: un área ambulatoria, un área de hospitalización y un área quirúrgica. Los servicios punteros en el mundo, con vocación académica, disponen igualmente de una cuarta área formada por laboratorios experimentales, tanto de fisiología humana, como de experimentación animal. 
El área ambulatoria suele estar constituida al menos por 4 laboratorios: electroencefalografía, electromiografía o sistema nervioso periférico, potenciales evocados y control motor. Algunos servicios disponen de laboratorios específicos de sistema nervioso vegetativo, dolor o microneurografía, en función del interés académico de los especialistas del servicio. En la mayor parte de los centros se incluyen al menos algunas de estas pruebas en los laboratorios de electromiografía o de potenciales evocados.

El área de hospitalización habitualmente esta formada por una unidad de vídeo-electroencefalogramas (vídeo-EEG) y una unidad de sueño. El número de habitaciones de las que constan las distintas unidades es variable en función del tamaño del hospital y del servicio. Las unidades de sueño y vídeo-EEG es aconsejable que sean polivalentes y se puedan emplear las habitaciones tanto para estudios polisomnográficos, como para monitorizaciones de larga duración mediante vídeo-EEG, ya sea de pacientes epilépticos con fines diagnósticos o quirúrgicos u otro tipo.

Cada día son más demandadas en neurofisiología clínica las monitorizaciones quirúrgicas. Su objetivo consiste en comprobar mediante diversas técnicas (estimulación cortical cerebral, potenciales evocados somatosensoriales, visuales, auditivos y motores, estimulación de raíces motoras, entre otras) la funcionalidad de las distintas estructuras nerviosas tanto centrales como periféricas durante intervenciones quirúrgicas con el fin de establecer su estado preoperatorio, tratar de preservar su indemnidad durante el acto quirúrgico y establecer un pronóstico postoperatorio. Las monitorizaciones son de muy distinta índole, ejemplos de algunas son: localización de la cisura de Rolando mediante potenciales evocados somestésicos en neurocirugía hemisférica, localización del área motora primaria, cápsula interna o área de lenguaje mediante estimulación eléctrica, localización del núcleo subtalámico u otros núcleos de los ganglios basales o talámicos para la colocación de estimuladores pro- fundos de cara a tratar la enfermedad de Parkinson, temblores u otros movimientos anormales, electrocorticografía con el fin de hacer resecciones a demanda en cirugía de la epilepsia, registro EEG durante cirugías vasculares con el fin controlar lesiones isquémicas, confirmación de la adecuada localización de implantes eléctricos en núcleos cocleares para tratamiento sordera por lesión bilateral retrococlear, monitorización de vías sensoriales o motoras en cirugía cervical, de escoliosis u otras cirugías, monitorización de la cola de caballo o de raíces y monitorización de la indemnidad de troncos nerviosos. Las unidades de monitorización intraoperatoria deben trabajar fundamentalmente en colaboración con los servicios de Traumatología, Neurocirugía, Otorrinolaringología, etc y su éxito es el adecuado entendimiento entre los distintos grupos.

El futuro de la Neurofisiología es prometedor y la especialidad se adapta al mundo tan rápidamente cambiante de la medicina. No tiene sentido quedar anclado en exploraciones clásicas, algunas con casi un siglo de antigüedad, que tarde o temprano quedaran desfasadas por otras tecnologías nuevas. Es importante por tanto que el especialista en Neurofisiología Clínica tenga una base sólida en neurociencias y un conocimiento exhaustivo de las enfermedades del sistema nervioso que le permita esta adaptación. El futuro viene de la mano de la aproximación multidisciplinar a los problemas. El neurofisiólogo tiene que aprender a trabajar con ingenieros, biólogos, radioquímicos u otros especialistas médicos. Existen abundantes técnicas que exploran la fisiología del sistema nervioso donde el neurofisiólogo tiene que tener un papel de relevancia en el futuro próximo. Un ejemplo es la resonancia magnética funcional (RMf). La RMf habitualmente se encuentra situada en los servicios de radiología pero en los centros importantes a nivel mundial los equipos son multidisciplinares participando: ingenieros, radiólogos, físicos, psicólogos, neurólogos o neurofisiólogos. La RMf es una exploración funcional del sistema nervioso y como tal el neurofisiólogo debe estar presente. La neurofisiología puede aportar la resolución temporal 
que le falta a estas técnicas de neuroimagen con gran resolución espacial. Hoy es posible en neurofisiología, y en Navarra lo tenemos disponible, realizar registros de EEG o potenciales evocados de alta resolución, con digitalización de electrodos y localizar sobre la RM del paciente, con los algoritmos adecuados para solucionar el problema inverso, el generador de una determinada actividad. Igualmente se puede coregistrar el EEG en la resonancia magnética con el fin de disparar la adquisición y ver la activación cerebral en un momento temporal, durante un evento concreto externo o cerebral. Por ejemplo, realizar la RMf cuando descarga una punta-onda en el EEG. De esta forma se pueden conocer las regiones activadas durante este grafoelemento paroxístico ${ }^{2}$.

La estimulación magnética con pulsos únicos o pareados es una técnica utilizada ya hace bastantes años con fines diagnósticos y en la que Navarra fue pionera en España. Desde hace años se está estudiando la posible acción terapéutica de la estimulación magnética repetitiva ${ }^{3}$. Se ha ensayando en distintas patologías como: la depresión, dolor, neurorrehabilitación u otros problemas neuropsiquiátricos. Los resultados han sido un poco variables, pero posiblemente en un futuro no lejano pueda utilizarse en la clínica diaria con fines terapéuticos, en alguna de estas patologías.

Un campo muy prometedor es el estudio de la actividad oscilatoria cerebral tal como se revisa en una de las aportaciones de este número ${ }^{4}$. Las oscilaciones cerebrales son un mecanismo básico del funcionamiento cerebral que surge fruto de interrelación de los múltiples elementos de una red neuronal. Distintas patologías tienen alteraciones en la dinámica de las redes neurales y de sus oscilaciones y pueden ser la causa de parte de la sintomatología. Fruto de la colaboración interdisciplinar, especialmente con ingenieros y físicos se van desarrollando herramientas matemáticas que permiten su análisis y hacen comprensible su papel fisiopatológico. Posiblemente en un futuro puedan tener un papel diagnóstico y puedan diseñarse acciones terapéuticas dirigidas a cambiar su comportamiento. Posiblemente el mecanismo de la acción terapéutica de la estimulación profunda en el tratamiento de la enfermedad de Parkinson sea interferir con las oscilaciones anómalas de los ganglios basales presente en estos pacientes ${ }^{5}$.

Dos campos que han experimentado un enorme crecimiento en la neurofisiología son los estudios del sueño ${ }^{6}$ y la monitorización neurofisiológica quirúrgica ${ }^{7}$. Es de prever que este crecimiento continúe en los próximos años y que otras técnicas como análisis cinemáticos de la marcha, movimientos oculares ${ }^{8}$, etc. se vayan sumando al abanico de pruebas complementarias de utilidad para el diagnóstico de las enfermedades neurológicas.

\section{BIBLIOGRAFÍA}

1. Boletín Oficial del Estado. Orden/SCO/2617/2008 de 1 de septiembre por la que se aprueba y publica el programa formativo de la especialidad de Neurofisiología Clínica. BOE núm 224, $16 / 9 / 2008$.

2. Gotman J, Kobayashi E, Bagshaw AP, Bénar CG, DUBEAU F. Combining EEG and fMRI: a multimodal tool for epilepsy research. J Magn Reson Imaging 2006; 23: 906-920.

3. Daskalakis ZJ, Levinson AJ, Fitzgerald PB. Repetitive transcranial magnetic stimulation for major depressive disorder: a review. Can J Psychiatry 2008; 53: 555-566.

4. Thut G, Miniussi C. New insights into rhythmic brain activity from TMS-EEG studies. Trends Cogn Sci 2009; 13: 182-189.

5. Hammond C, Bergman H, Brown P. Pathological synchronization in Parkinson's disease: networks, models and treatments. Trends Neurosci 2007; 30: 357-364.

6. Chocroverty S. Sleep Disorders Medicine: Basic Science, Technical Considerations and Clinical Aspects. 2 edition. Boston: Butterworth-Heinemann, 2000.

7. Deletis V, Sala F. Intraoperative neurophysiological monitoring during spine surgery: an update. Curr Opin Orthop 2004; 15: 154-158.

8. Lencer R, Trillenberg P. Neurophysiology and neuroanatomy of smooth pursuit in humans. Brain Cogn 2008; 68: 219-228. 